\title{
Identification Filtering with fuzzy estimations
}

\author{
J. J., Medel J ${ }^{1}$ \\ ${ }^{1}$ Computing Research Centre \\ Venus St., Tel: 56242000 ext. 56570 \\ CP. 07738 Mexico D. F
}

\author{
J. C: Garcia I ${ }^{2}$ \\ ${ }^{2}$ Mechanical and Electrical Engineering School \\ Santa Ana Av., Tel: 56242000 ext. 73258 \\ Mexico D. F
}

J. C. Sanchez $\mathrm{G}^{3}$

\begin{abstract}
A digital identification filter interacts with an output reference model signal known as a black-box output system. The identification technique commonly needs the transition and gain matrixes. Both estimation cases are based on mean square criterion obtaining of the minimum output error as the best estimation filtering. The evolution system represents adaptive properties that the identification mechanism includes considering the fuzzy logic strategies affecting in probability sense the evolution identification filter. The fuzzy estimation filter allows in two forms describing the transition and the gain matrixes applying actions that affect the identification structure. Basically, the adaptive criterion conforming the inference mechanisms set, the Knowledge and Rule bases, selecting the optimal coefficients in distribution form. This paper describes the fuzzy strategies applied to the Kalman filter transition function, and gain matrixes. The simulation results were developed using Matlab ${ }^{\oplus}$.
\end{abstract}

Keywords- Intelligent Identification; Digital identification filter; Fuzzy estimation; Signal processing; Probability.

\section{INTRODUCTION}

The intelligent inference mechanisms are built and characterized with respect to dynamical system operation uncertainties bounded by a distribution function having different operational levels and needing correct answers in accordance to their dynamic changes. The adaptively is the condition needed in digital systems that interact with the real processes. The identification filter describes the natural evolution based on objective functions adjusting their parametres and gains giving the correct solution for a time interval. With this perspective, one of the best tools used to solve the filter identification is a recursive digital filter with the adaptive parametre and gain estimation giving a limited temporal answer integrated both in the identification frame. The problem observed in the Kalman filter is that it supposes known internal black box parametres and described the gain matrix. Unfortunately, the adaptability of it to natural reference system changes is lost. The unknown parametres and gain are characterized by inference mechanisms in accordance to different dynamical operation levels, corresponding to a fuzzy system description, with different rank operations [1], [2], [3], [4], [5], [6]. The paper integrates the fuzzy model concepts as a intelligence system that describes with time restrictions the Kalman transition and gain adjusting the answers in a natural form to the reference process output answer [7], [8].
Digital fuzzy filter developed as parametre and gain estimations operating automatically into identification improving the identification filter performance changes. The operational fuzzy tools are the inference mechanisms selecting into the Knowledge Bases (KB) the best parametre and gain with respect to the Identification Error Density (IED). These results are applied to the conventional Kalman SISO filter permitting it to be transformed into an expert identification filter in spite of environmental changes suffer by the reference output system [9].

\section{FUZZY ESTIMATION FILTERING}

A fuzzy estimation filtering operates in a close loop with: a) Transition function considering the fuzzy logic identification density error in adaptive parametre estimations and, $b$ ) The Kalman gain with respect to identification density error, c) The density identification error ranks with variable stages considering that the identification error variable through the time. Commonly the estimation filters do not use the operational level processes with time restrictions [3,] [10] satisfying the identification filter requirements. The adaptive inference mechanism regions are built as Identification density error functions [11], being the first stage required in the fuzzy filter in accordance to a knowledge base membership function used in inference rules with adaptive boundaries. Fig. 1 in the diagram block scheme shows the process observing the transition function and gain described as parametres filter estimation $\hat{a}(k)$ and $K(k)$ affecting directly the identification Kalman answer $\hat{y}(k)$ based on the desired signal $y(k)$ (output reference process), generating the identification error $e(k)$ and also affecting the estimation and gain processes. The estimation filtering process dynamically adjusts its parameters in accordance to the membership value selected from the Knowledge Bases appropriates values that allow that the Kalman output signal converges to reference [12]. The estimation fuzzy filter in agreement with [4], [13] have the following elements:

1. Input inference: A desired signal and the identification error as the inputs.

2. Rules base: The expert membership functions (parametre and gain values) are updated into identification filter [14]. The membership functions metrics are dynamically 
adjustable considering the distribution identification error $e(k)$, using the logical connector if.

3. Inference mechanism: The expert actions select the parametre and gain in accordance to objective functions into specific membership from the Knowledge Bases respectively using the logical connector then selecting the section in where the parametre and gain adequate adjust the identification filter [8].

4. Output inference: This is the result the estimation fuzzy filter emits to be used into the Kalman identification filter in accordance with the reference signal process.

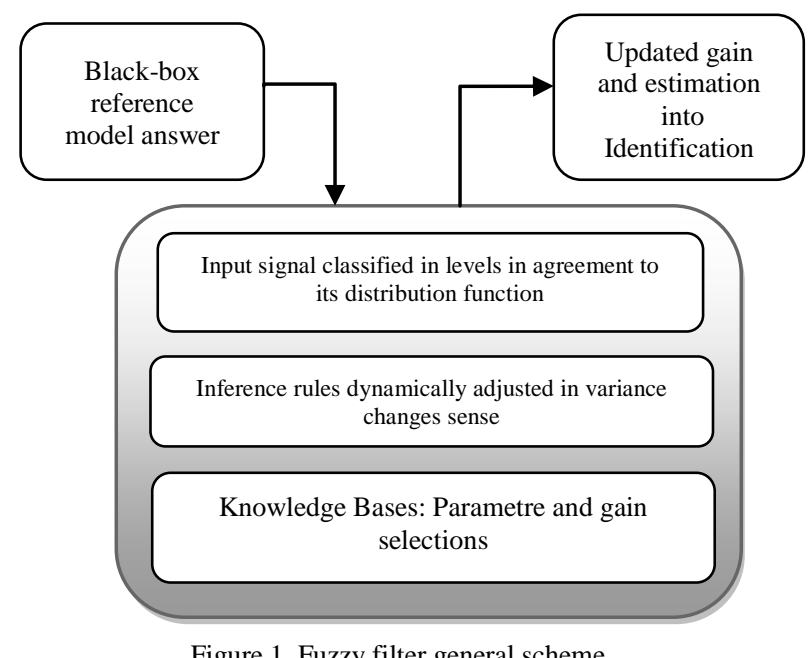

Figure 1. Fuzzy filter general scheme

\section{FUZZY ESTIMATION AND KALMAN FILTERING PROPERTIES}

A fuzzy estimation adjoins into Kalman filter considers the stochastic digital filter properties and the distribution functional error classified in intervals and associated with intelligence decisions affecting the identification filter [3]. Fig. 2 shows the filter architecture integrating the fuzzy estimation scheme.

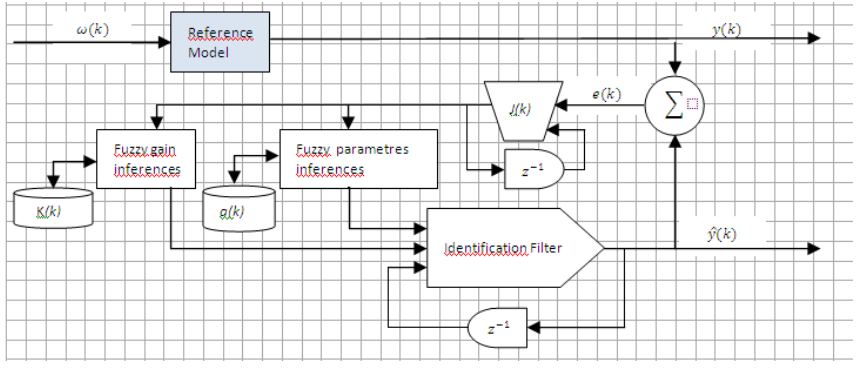

Figure 2. Identification Filter with fuzzy filter estimations

Fig. 2, showed the fuzzy stages needed by the identification filter: $x(k)$ is the reference model input, $y(k)$ is the reference model output and the fuzzy filter input, $\hat{a}(k)$ is the fuzzy parametre value, $K(k)$ is the fuzzy gain, $e(k)$ is the error, $J(k)$ is the functional error and $\hat{y}(k)$ is the identification filtering output.
Rule base: The new information arriving at the estimation filter in accordance to the reference signal changes describing the operational levels [8]. The fuzzy rules base has a set of logical connectors (if-then) classifying the reference process conditions in levels, selecting in probability sense the best membership value from the Knowledge Bases in order to update these into the identification filter. This mechanism is limited by the error filter criteria, which previously had all the possible parametre and gain values as a membership function in the Knowledge Bases, in accordance with the reference model interaction [4]. The logical connectors (if-then) allow selecting the operational levels membership function indicators (parametre $\hat{a}(k)$ and gain $K(k)$ values) adjusting the identification filtering process [14].

The fuzzy mechanism has the following description [9]:

Inference mechanisms use if connector, in order to find the reference model operational levels considering the identification error value in accordance to fuzzy mechanism assigning the membership function then connector. This membership value selects from the Knowledge Bases the parametre an gain values affecting the identification filter $\hat{y}(k)$. The fuzzy filter estimation uses second probability moment recursively (1) considering that the identification error has stationary conditions and it is bounded in levels [4]. $J(k)=\frac{1}{k}\left[e(k)^{2}+(k-1) J(k-1)\right] \in R_{[0,1)}$

The fuzzy mechanisms make the minimization error described as $J_{\min }$, having the best neighborhood value to the desired signal $y(k)$. The error must be near to $\gamma$ within the interval limit $[0,1)$. The filter membership function answer levels have an affinity between the minimum and maximum intervals limiting the operation description inside the filter distribution function [2], [12]. Fig. 3, shows the filter representation criterion describing its convergence.

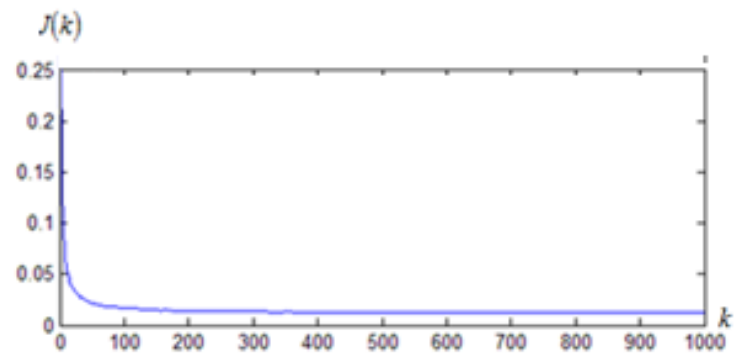

Figure3. Filter convergence functional error (1).

Fig. 3 shown the dynamical error minimization based on the stochastic gradient criterion classified in error levels and in fuzzy stages having the best neighborhood desired values (parametre and gain), in order to update the new conditions required into identification scheme [11].

\section{A. Parametre and gain selection}

The membership function selects the parametre $\hat{a}(k)$ and gain values $K(k)$ into the fuzzy stage in accordance with the 
reference model operational levels and inference rules developed in probability sense where the Knowledge Bases are bounded by a control area $T_{N}$ as [4]:

$$
T_{N}=\{(y(k), \hat{y}(k))\} \subseteq R^{2}
$$

As an example, the membership functions set into the parametre Knowledge Base is bounded by the error criterion [8], [9]. Fig. 4, shows the dynamical filter parametre estimation $\hat{a}(k)$, in accordance with the reference model evolution $y(k)$ and its identification $\hat{y}(k)$.

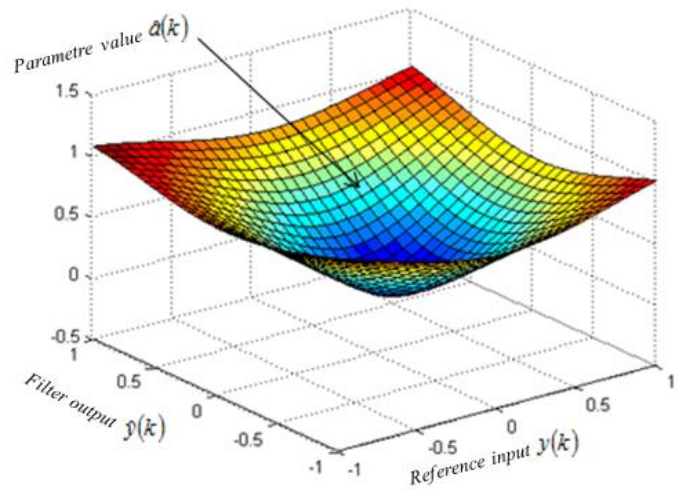

Figure 4. Knowledge Base viewed as a parametric surface.

Fig. 4 shown the parametre selection into operational levels (2) based on changes into the filter input-output conditions [7].

- The parametre and gain selections goal using fuzzy logic strategies [12], [14]:

- Automatic filter parametre and gain selections base on different levels.

- Parametre and gain selections in accordance with the desired signal and the identification filter response.

- Adaptive rules modifying the membership functions limits, renewing and updating its values in accordance to the reference model and the error criteria

- Each membership value establishes the maximum correspondence between the desired and the reference signals, minimizing the identification filter error [2].

\section{B. Time constrains}

Identification integrated with fuzzy parametre estimation $\hat{a}(k)$ and gain $K(k)$ strategies is bounded temporally, considering the quality response in accordance with filtering stability and time restrictions [15]. Global, are described by functional identification error (see Fig. 4).

If the identification error is stationary then the maximum convergence rate is limited to minimum region established as an identification variance error function. The convergence intervals are $[0, \varepsilon \pm \alpha)$ with functional error $J(k)$ upper limit tending to zero. The membership functions are temporarily bounded by (3). The global characteristics are specified in probabilistic form where $J\left(\tau_{m}\right)=\inf \left\{\min \left\{\tilde{J}_{k}\right\}\right\} \leq \varepsilon$ and $\left\{\widetilde{J}_{k}\right\} \subseteq\left\{J_{k}\right\}$ considering $P\left(\widetilde{J}_{k} \leq \varepsilon \pm \alpha\right)=1$ in accordance with the reference model [4].

$$
\tau_{\min }=0.5 f_{\max }^{-1}
$$

The final filter times $f(k)_{i}$ inside its corresponding absolute limits $\left[l d(k)_{i_{-} \min }, L D(k)_{i}\right)$ in the Nyquist and Shannon sense [15], the filter integrate time is limited dynamically considering that $\left.\mu \mid l d(k)_{i_{-} \min }, L D(k)_{i}\right)<\tau_{\min }$. Fig. 5, shows the time interval filter process task [4].

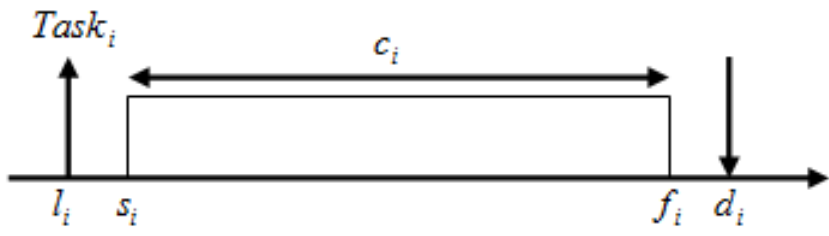

Figure 5. Filtering process task,

The filtering process task is bounded by (3) having the arrival time $\left(l_{i}\right)$, start time $\left(s_{i}\right)$, processing time $\left(c_{i}\right)$, final time $\left(f_{i}\right)$ and the maximum time period $\left(d_{i}\right)$.

\section{RESULTS}

Identification technique considers the fuzzy filter estimation describing the known internal black-box parametre and the identification gain (see Fig. 2). Reference model considered an Autoregressive Mobil Average (ARMA) model interacting with fuzzy filter. The discrete SISO states space system as reference model is shown in (4) and (5).

$$
x(k+1)=a(k) x(k)+w(k)
$$

The filter output is described in (5) as $\operatorname{ARMA}(1,1)$ model:

$$
y(k)=c(k) x(k)+v(k)
$$

With $\quad x(k) \in R,|a(k)| \in R_{[0,1)},\{w(k)\} \subseteq N\left(\mu_{w}, \sigma_{w}^{2}<\infty\right)$, $y(k) \in R,|c(k)| \in R_{[0,1)},\{v(k)\} \subseteq N\left(\mu_{v}, \sigma_{v}^{2}<\infty\right)$, where: $\mathrm{x}(\mathrm{k})$ is the internal reference model state; $\mathrm{a}(\mathrm{k})$ is the parametre; $\mathrm{w}(\mathrm{k})$ is the reference model noise; $\mathrm{y}(\mathrm{k})$ is the reference model signal as a filter input; $\mathrm{c}(\mathrm{k})$ is the parametre output system and $\mathrm{v}(\mathrm{k})$ is the output vector noise.

The different operation levels must match with the error criteria established in accordance with the desired signal $y(k)$; the parametre $a(k)$ and gain as $K(k)$ selections from the Knowledge Bases sand the filter response $\hat{y}(k)$. The first step in accordance with the dynamical selection into the identification $\operatorname{ARMA}(1,1)$ model is described in (6) .

$$
\hat{y}(k)=\hat{a}(k) \hat{y}(k-1)+\hat{W}(k)
$$

Fig. 6 shows the desired signal $y(k)$ and its approximation to the identification filter response $\hat{y}(k)$. 


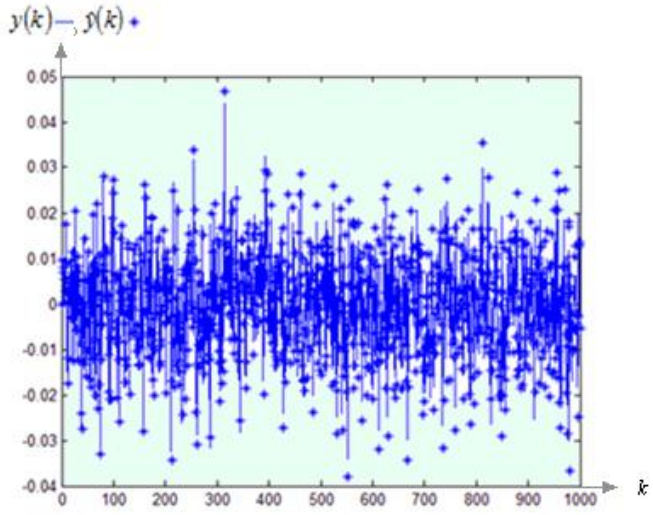

Figure 6. Desired and signal approximations.

In accordance with the reference model operation, the adaptive fuzzy process stage uses the inference mechanisms, selecting the dynamically the parametre value in agreement to (5). Fuzzy inference with respect to the error levels limit the rules set. Fig. 7 shows the error classification in order to select the best answer.

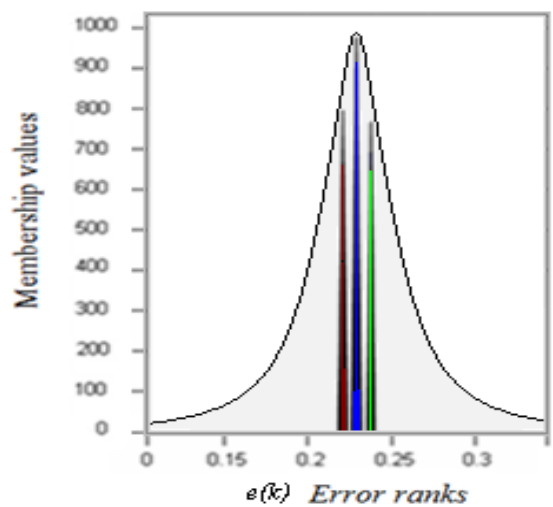

Figure 7. Error levels Classification.

In accordance with the estimation fuzzy filter into the selection process, Fig. 8 shows different filter response operational levels $\hat{y}(k)$.

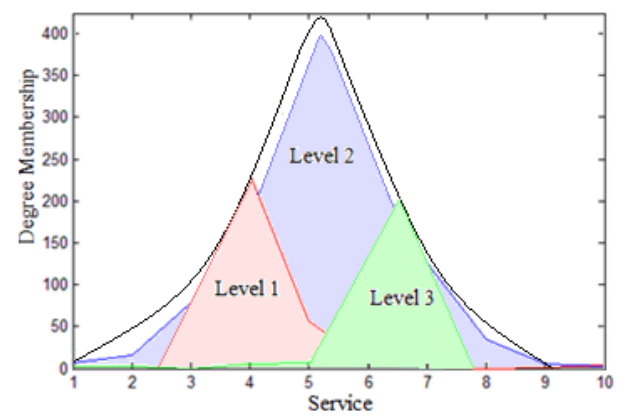

Figure 8. Filter degree operational levels process.

The distribution functions of reference output model and its identification converge in distribution sense viewed in Fig. 9. The identification filter answer describes the reference signal observing in Fig. 9, that in distribution; second converge to the desired answer, selecting into the knowledge base the best parameter, without permits that the filter response breaks the border.

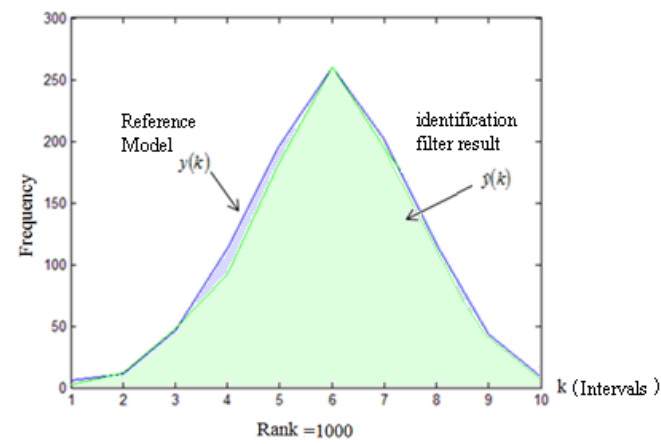

Figure 9. Identification filter bounded by the reference model, in distribution sense.

\section{ACKNOWLEDGMENT}

Thanks to Prof. Lorenz Whitehill Waterson (Native English Speaker), National Polytechnic Institute (IPN) and National Council of Science and Technology (Conacyt) for their support, to make this work possible.

\section{REFERENCES}

[1] Abdul, A. Fuzzy Logic, Imperial College of London, (2003).

[2] Ash, R. Real Analysis and Probability, E.U.A. Ed. Academic Press, (1970).

[3] García, J., J. Medel and J. Sánchez, Filtrado Neuronal Difuso: Caso MIMO, Revista Ingeniería e Investigación, Vol. 31 (No. 1), (2011).

[4] García, J., J. Medel and P, Guevara, Filtrado Digital Difuso en Tiempo Real, Revista Computación y Sistemas, Vol. 11 (No. 4), (2008).

[5] Haykin, S, Adaptive Filtering, E.U.A., Prentice Hall, (2001).

[6] Leydesdorff, L, The Construction and Globalization of the Knowledge Base in inter-human communication systems, Canadian Journal of Communication, Vol. 28, 267:289, (2003).

[7] Mollov, S., R. Babuska, J. Abonyi, and H. Verbruggen, Effective Optimization for Fuzzy Model Predictive Control, IEEE Transactions on Fuzzy Systems, Vol. 12, 661:675, (2004).

[8] Zadeh, L. The Concept of a Linguistic Variable and its Applications to Approximates Reasoning, Information and control, Vol. 8, 338:353, (1965).

[9] Schneider, M. and A. Kandel. Fuzzy expert systems tools, Wiley, Inglaterra, (1996).

[10] Margaliot M. and G. Langholz, New Approaches to Fuzzy Modeling and Control Design and Analysis, World Scientific, Israel, (2000).

[11] Mamdani, E, Applications of Fuzzy Algorithms for Control of Simple Dynamic Plant, Proc. IEEE, Vol.121, 1585:1588, (1974).

[12] Medel, J., J, García and J, Sánchez, Real-time Fuzzy Digital Filters Properties for SISO Systems, Automatic Control and Computer Sciences AVT, Vol. 42 (No. 1), 26:34, ISSN: 0146-4116, (2008).

[13] Passino, K. M. Fuzzy Control, E.U.A., Addison Wesley, (1998).

\section{AUTHORS PROFILE}

Dr. Medel, is a Prof. working from 1998 into Computing Research Centre. He has 3 patents, more that 51 international papers indexed. He consider that the fuzzy logic model descriptions is very important in the real life. Actually the Prof. collaborates with others researchers in Real-time and identification theory.

Dr. Garcia develops Associate Profesor activities with resect to filter theory using fuzzy logic tools. Actually, he has one graduated, and 10 indexed papers.

Dr. Sanchez is a Prof., interested in identification and filter systems with time restrictions. He has more than 74 indexed papers and more that 34 graduated. 